\title{
L'EPOPEA DI UN UOMO SENZA QUALITÀ: KLIM SAMGIN
}

\author{
ERICA KLEIN
}

ABSTRACT. - Nel suo ultimo romanzo fiume, La vita di Klim Samgin (1924-1936) rimasto incompiuto, Gor'kij si estranea dalle problematiche del nuovo corso sovietico. Lontano dal realismo socialista sembra piuttosto inserirsi nello spirito del nuovo romanzo europeo (Thomas Mann, Musil), dimostrando di trovarsi perfettamente a suo agio con le rivelazioni dell'inconscio, le teorie dello spazio psicologico, il flusso della coscienza.

Grande epopea delle idee, quest'opera testamento guarda a ritroso, ripercorrendo le suggestioni, le passioni, le ossessioni che hanno occupato le menti dell'intelligencija russa nei 40 anni precedenti la rivoluzione d'ottobre. Al centro: un personaggio meschino (storia di un'anima vuota è il sottotitolo del romanzo), bloccato nella sua crescita emotiva da antiche nevrosi irrisolte. E' un uomo che inventa se stesso, convinto che basti un buon sistema di frasi a fare l'uomo.

La forza delle ideologie offre comodo riparo alla pochezza spirituale dell'uomo mediocre che si affaccia alla storia e ritiene di poter continuare a tessere la propria rete di grettezze quotidiane sotto l'imperio e l'ipnosi di formule assolute.

$$
* * *
$$

В своём последнем незаконченном безконечном романе “Жизнь Клима Самгина" (1924-1936) Горький удаляется от проблем нового советского курса. Далёкий от социалистического реализма он, скорее всего, приближается к духу нового европейского романа (Т. Манн, Музил), где чувствует полную свободу в проявлениях подсознания.

Это произведение - завещание - огромная эпопея идей, занимавших русскую интеллигенцию за 40 лет до революции. В центре - ничтожный герой, который не смог эмоционально развиваться из-за перешённых проблем, связанных с неврозом. 
Этот человек придумывает сам себя, он убеждён в том, что хороший набор фраз может заменить человеческую личность, так как в мире идеологий вполне можно продолжать делать свои мелкие дела, прикрываясь высокой фразой.

Uno dei tanti paradossi che accompagnano la figura di Gor'kij è quello di aver scritto un romanzo della coscienza in epoca di consolidamento del realismo socialista di cui, oltretutto, era considerato il patrono. Un romanzo con cui Gor'kij si affaccia alla modernità europea del '900, prendendo atto della rivolta individualista che dilaga ovunque in letteratura e del fatto che alla realtà intesa tradizionalmente come fatto inequivocabile si è sostituita una realtà variabile prodotta dalla visione deformata del singolo. E' l'epoca delle grandi tentazioni, dell'uomo che vuole osare essere oltre i valori intesi come dati immutabili, che crede sia giunto per lui il momento di scompigliare i concetti accreditati di bene e di male, e di riformularli in modo nuovo, a seconda della percezione che se ne ha e dell'utilità che ne deriva. Nulla più è stabile, la vita scorre come uno sterminato intreccio di elementi e di sensazioni che si è liberi di collegare in proprio e di chiamare sostanze.

Il nuovo tipo di romanzo europeo che si fa strada tiene conto di questa voglia di sfaldamento e riproposizione svincolata da norme, e pone ormai al centro dell'universo l'interiorità di un individuo, non necessariamente speciale, ma sempre così importante da farsi, per l'intero corso della narrazione, mediatrice fra l'Io e il mondo.

E' con queste premesse che ho preso in prestito da Musil la definizione di "uomo senza qualità" per Klim Samgin e non perché i due protagonisti si assomiglino - anzi, sotto questo profilo, la mia operazione è stata perfino abusiva - ma per un'analoga disposizione che mi è parso di cogliere in entrambi, a relativizzare ogni problema, a tentare di liberarsi dall'assalto della realtà solo con le parole, e anche per una sorta di inerzia dell'anima che li costringe a farsi spettatori della loro vita, senza poter scegliere alcunché: Ulrich per indecisione, Klim per pochezza di spirito, entrambi per impedimenti interni, a loro stessi poco chiari.

Gor'kij e Musil hanno avuto in sorte la comune esperienza di vivere, negli stessi anni, il declino di società che presagivano la loro fine, senza immaginare che sarebbe potuto succedere davvero, e di scriverne quando le loro civiltà di riferimento, Russia zarista e Impero asburgico, erano crollate da poco, e in maniera così precipitosa e definitiva da non lasciare spazio ad alcuna strategia di elaborazione difensiva. 
Il loro viaggio a ritroso, divenuto in entrambi i casi grande monumento di riflessione, di critica, di ironia, di nostalgia (quel mondo scomparso e, dopo tutto rimpianto, non aveva ormai altro luogo dove sopravvivere se non in letteratura), ha anche il carattere di ricerca delle ragioni occulte, autodistruttive, oltre che storiche e culturali, di quello sprofondamento e dell'incredibile dopo che aveva osato presentarsi. In entrambi, i simboli del tempo di cui erano vissuti si erano fatti comprensibili solo più tardi e avevano richiesto un'elaborazione anche psicologica di ciò ch'era avvenuto.

Ciò che allontana Gor'kij da Musil è il diverso rapporto che intrattiene con il suo protagonista. Se per Musil si può parlare di attitudini convergenti, di affinità di pensiero e di sentire, nel caso di Gor'kij è esclusa qualunque sovrapposizione. Gor'kij non amava il suo personaggio e questo è il grande mistero dell'opera. Perché scegliere come interlocutore della sua ultima grande avventura un personaggio così sgradevole, lui davvero un uomo senza qualità, senza qualità umane, senza generosità, senza calore (Ulrich è tutt'altro invece, pieno di qualità, anche se queste sembrano quasi esistere senza di lui, senza un centro che le possa coordinare e utilizzare, qualità senza l'uomo).

La critica sovietica tradizionale su questo problema se la cava con la consueta sbrigatività: l'intento di Gor'kij sarebbe stato mostrare la miseria spirituale dell'intellettuale borghese che non si è schierato in tempo e apertamente per la rivoluzione. Il suo critico più famoso, A. L. Ovčarenko, dice che uno come Samgin poteva scomparire solo con la scomparsa della società che l'aveva generato. Non è così semplice e neppure vero. Per quanto strano possa sembrare, la problematica di Gor'kij non è tanto sociale, quanto esistenziale. Al centro di questa sua nuova indagine è ancora una volta la personalità umana, il suo radicamento in una zona irrazionale della psiche, non del tutto raggiungibile dall'ambiente, dalla classe, dall'ideologia. C'è dell'altro nell'uomo, qualcosa di indistruttibile, di non pianificabile, di legato al grado di emotività del suo pensiero. Riteneva come Foma Gordeev, protagonista del suo primo romanzo, che ogni uomo, come la terra, fosse avvolto dalla sua particolare atmosfera, che dipendeva dalla sua energia, dal fuoco di cui disponeva la sua anima. Klim in realtà, non è inquadrabile in un tipo sociale ben definito, da vero personaggio artistico ha qualcosa di più universale. Non c'è nessuno in fondo, che non possa riconoscersi almeno un pochino in lui. Samgin non è nessuno, ma è ciascuno, è la tentazione verso la bassezza che, a tratti, a volte alberga in ognuno 
di noi. Del resto non sembra credibile che Gor'kij abbia convissuto con Samgin per dodici anni e migliaia di pagine per un progetto di così poco respiro e propaganda antiborghese. E' più credibile l'accentuarsi di un interesse personale ad approfondire temi psicanaliticamente cruciali della natura umana, temi che oltretutto erano da tempo al centro della sua attenzione: la doppiezza, il camuffamento, l'alienazione, il silenzio della coscienza, temi che, alla luce delle nuove catastrofi, chiedevano evidentemente una più attuale investigazione.

C'era stato Karamora negli anni '20 a testimoniare la propensione di Gor'kij per questo tipo di analisi, un delatore, passato senza problemi di coscienza dai circoli rivoluzionari all'Ochrana. Alla fine, scoperto, finito in prigione e in attesa dell'esecuzione, scrive un lungo diario, cercando di venire a capo delle proprie oscure pressioni interne, dei propri comportamenti inqualificabili: “...Avevo fatto il doppio gioco, così, per variare, per vedere cosa sarebbe successo, per punzecchiare la coscienza, provare a risvegliarla. Sentivo che dentro di me viveva qualcuno, un ospite indesiderato e sgradito che ascoltava i miei discorsi e mi osservava con diffidenza....ma la coscienza taceva". ${ }^{1}$

Ancor prima, nel 1908, c'era stato Klimkov in La vita di un uomo inutile, piccolo orfano che diventa, suo malgrado, spia zarista, anch'egli alle non facili prese con i propri contrasti, ma Samgin, il più intellettuale, analitico, raffinato dei doppiogiochisti, è anche il più inconsapevole, aiutato, nelle sue rapide manovre di razionalizzazione, proprio dal ricco bagaglio culturale di cui dispone e dalla prontezza dell'agile intelletto. Il suo doppio gioco è infatti esclusivamente con se stesso. Nessuno lo accusa, nessuno lo incalza, nessuna situazione esterna lo mette alle strette, egli è unicamente impegnato a discutere con se stesso per trovare argomenti sempre nuovi, sempre validi, sempre adeguati al mantenimento del proprio stato di quiete morale. Da ogni dibattito interno emerge puntualmente la sua indiscutibile inattaccabilità.

Da dove viene fuori questo eroe negativo del secolo, il più banale e opportunista degli uomini superflui? Potrebbe sembrare un romanzo di formazione questa Vita di Klim Samgin, ${ }^{2}$ si seguono infatti tutte le tappe del protagonista fino al suo affermarsi professionalmente come

1. M. Gor'kij, Karamora, in Gor'kij - Opere scelte, Editori Riuniti, Roma 1980, vol. 10, pag. 192.

2. I primi tre volumi di La vita di Klim Samgin (quarant'anni) uscirono contem- 
avvocato di successo. Ma è un abbaglio, si tratta piuttosto di una formazione non riuscita, rimasta bloccata dall'impossibilità tutta interiore e psicologica di attuare uno scambio proficuo con la realtà fuori di lui. Rimane emotivamente estraneo agli avvenimenti e alle persone che lo incrociano, prigioniero di un modello di rapporti umani che gli impedisce di vedere negli altri qualcosa di buono e quindi di arricchirsene.

Lo scenario dove si produce e avanza questa malattia dell'anima è di larghe ampiezze temporali, da qui la denominazione di "epopea" spesso attribuita al romanzo. Ma si tratta più che altro di un'epopea delle idee, piena zeppa com'è di tutto ciò che si è pensato, detto, dibattuto nei 40 anni di vita russa che vanno dalla morte di Alessandro II agli albori della rivoluzione d'ottobre. Periodo in cui le suggestioni culturali erano tante, e delle matrici più diverse, dai populisti ai marxisti, ai decadenti, ai mistici, ai tolstojani, ai settari, a quant'altro, e dove i temi erano quelli massimalisti di sempre, di tutto l'800: il destino della Russia, la sua missione, la sua identità, la sua parola da dire al mondo, la sua alterità rispetto all'Occidente. Ma Gor'kij non intende proporre cartelli ideologici, né perorare alcuna causa, il romanzo è totalmente antiideologico, impossibile perfino identificare la sua personale posizione e se un giudizio prevale sugli altri è quello critico sulla tirannia delle idee dietro i cui proclami trovano comodo riparo meschinità varie, rivalse, risentimenti, invidie. Un nuovo uomo del sottosuolo versione ' 900 si fa avanti, con una facilitazione: quella di poter contare sulla retorica ben congegnata delle formule mistificanti che mirano a ipnotizzare l'ascoltatore, a paralizzarne la volontà. Klim Samgin è il nuovo antieroe che si affaccia alla storia, l'intellettuale mediocre e conformista che si affida ai trucchi linguistici per nascondere la propria pochezza e farsi strada nella vita. E' l'opportunista moderno, privo di passioni, che avverte l'arrivo delle ideologie, del loro imperioso potere e si premunisce per tempo. Non cerca vantaggi materiali, ma ruba parole, perché nel regno dell'astrazione, sono loro la nuova, vera ricchezza.

poraneamente a Mosca e a Berlino, due nel 1928, il terzo nel 1930, il quarto, incompiuto e frammentario, fu pubblicato postumo nel 1937, a cura della Commissione del comitato centrale del partito, incaricata di raccogliere il carteggio e l'eredità letteraria di Gor'kij. La traduzione italiana delle prime tre parti (la prima uscita già nell'agosto 1931 e commentata da Leone Ginzburg sulla rivista "La Cultura") è stata curata da Erme Cadei per conto di Mondadori, ripresa da Einaudi e da Editori Riuniti. La quarta parte, tradotta da Filippo Frassati, è uscita nel 1962 presso Editori Riuniti. 
Vengono in mente alcune osservazioni di Lichačëv sulla demagogia: "Già negli anni Venti" - scrive nelle sue memorie La mia Russia "il potere delle formule verbali finì per occupare una posizione di rilievo. Il potere delle parole diventò la manifestazione più greve della cattività spirituale".

Ecco, Gor'kij fa iniziare molto prima questa tendenza, la fa coincidere con l'epoca di preparazione della rivoluzione, soprattutto quella del 1905, quando la pressione dell'idea sociale era così marcata da far sentire in colpa chiunque non scaricasse sull'autocrazia zarista il peso di ogni malessere esistenziale. "Il senso della vita dell'intelligencija prerivoluzionaria era tutto nella rivoluzione." - scrive a questo proposito Semën Frank in Krušenie kumirov - "Dubitare della realtà del materialismo o del socialismo significava tradire il popolo". ${ }^{4}$

Gor'kij inizia a scrivere il suo romanzo nel 1924 a Sorrento, dove finalmente ha trovato equilibrio e concentrazione dopo gli sconvolgimenti della rivoluzione, le liti con i bolscevichi, espresse con virulenza inaudita nei 77 Pensieri intempestivi di Novaja Žizn', dopo l'uscita dall'Urss nel 1921 per insistenza di Lenin, dopo i soggiorni in Cecoslovacchia e Germania, dove il rapporto con gli émigrés si era rivelato altrettanto poco praticabile di quello con i nuovi dirigenti sovietici.

Ma non può essere un caso che Gor'kij trovi la forza e il coraggio di intraprendere questa resa dei conti con l'intelligencija russa prerivoluzionaria e con tutto ciò che ne aveva occupato le menti, solo alla morte di Lenin. Una condizione di libertà improvvisa di cui non avrebbe potuto disporre senza questa perdita. Difficilmente Lenin avrebbe tollerato un nuovo romanzo dell'amico Gor'kij, l'unico scrittore di estrazione veramente popolare su cui potesse contare, incentrato sulle peripezie mentali di un'anima vuota, proprio mentre l'Urss cercava nella letteratura un puntello di costruzione di consenso, uno strumento di rieducazione. E la libertà che qui Gor'kij si prende è davvero grande, addirittura quella di non parteggiare per la rivoluzione, lui che secondo la mitologia ufficiale era impastato con la rivoluzione fin dalla nascita.

Le voci che compaiono nel romanzo sono davvero tante, i perso-

3. D. Lichačëv, La mia Russia, Einaudi 1995, p. 195.

4. S. Frank, Krušenie kumirov, in Russkaja Ideja v krugu pisatelej I myslitelej russkogo zarubežja, Isskustvo, Mosca 1994, vol. 1, p. 136. 
naggi più di 800 (tutti hanno diritto di parola e diritto a non essere derisi, né interpretati tendenziosamente), ognuno si scelga la verità che gli è più congeniale, sembra suggerire Gor'kij, non è qui il punto, non da questo dipende il suo essere uomo. Personaggi che entrano ed escono senza che un intreccio richieda la loro presenza. Un suo critico di oggi, Aleksandr Etkind, ${ }^{5}$ dice che quest'opera di Gor'kij assomiglia alla sua villa italiana, quella di Sorrento dove appunto scriveva di Klim Samgin. Proprio come quella casa, in cui abitavano persone d'ogni tipo ed estrazione sociale, parenti, amici, conoscenti di conoscenti e perfetti sconosciuti, il romanzo è pieno di individui, uomini e donne senza specifico contesto, né relazione reciproca, venuti unicamente a dire la loro, a testimoniare di se stessi.

Gor'kij così netto, tranciante, ripetitivo nella pubblicistica di quegli stessi anni (ricordo che la stesura della Vita di Klim Samgin si è protratta dal 1924 al 1936, anno della morte ed è rimasta incompiuta), qui si è preso una rivincita, ha voluto essere solamente artista. Di più, questo romanzo sembra essere l'altra faccia delle sue prese di posizione ufficiali. Se una simpatia s'impone decisa non è né per Kutuzov, il futuro bolscevico, indisponibile già da subito a qualunque compromesso con altre idee, né per gli altri possessori di verità come lui, ma piuttosto per la saggia, bellissima, scintillante protagonista femminile, Marina Zotova. Potente incarnazione dell'anima femminile, è l'unico personaggio completo del romanzo in grado di contagiare e di attirare possibili identificazioni. E' l'unico veramente consapevole delle proprie affermazioni e capace di cogliere qualcosa dell'essenza profonda della vita, fuori da teorie e griglie ideologiche. Pur immersa nel clima di generale rifiuto di ogni autorità che ha caratterizzato l'epoca dell'ultimo zar, pur aiutando finanziariamente gruppi di rivoluzionari, li considera fuori dal mondo. Di loro dice: "Sono come i vecchi credenti, si sono inventati un reame favoloso perché hanno paura di vivere". ${ }^{6} \mathrm{Ma}$ era forse proponibile affidare l'unico, autentico messaggio di salvezza, alla vigilia della rivoluzione, a questa giovane, esuberante, ricca vedova che commerciava in arredi sacri e svolgeva, in segreto, il ruolo di madonna (bogorodica), nocchiero di un'antica setta di flagellanti (chlisty)? Eppure è così. La

5. A. Etkind, Gor'kij i Bezbedov, podtekst Serebrjanogo golub'ja v Klime Samgine, in "Literaturnoe novoe obozrenie", n. 24, 1997.

6. M. Gor'kij, La vita di Klim Samgin, Einaudi 1955, Vol. 2, p. 1340. 
terza parte del romanzo è quasi interamente occupata dalla sua estesa presenza. E' lei la vera figura di capo carismatico, l'interprete delle istanze profonde dell'anima popolare simboleggiata qui dalla comunità dei settari, è lei l'unica capace di governarne anche l'elemento dionisiaco, la stichija, mai disgiunto dal bisogno spirituale di realizzare il regno di Dio sulla terra. Al suo confronto i vari intellettuali del romanzo sono pallide comparse disincarnate che fantasticano di teorie da applicare al corpo vivo dell'umanità, senza conoscerne la sostanza. Se pensiamo che questa parte del romanzo è stata scritta negli ultimi anni venti e continuamente interrotta dai ripetuti viaggi in Urss dove andava in onda la collettivizzazione forzata, è possibile farsi un'idea di ciò che pensasse Gor'kij degli eventi di casa e da quali altre idee fosse occupata la sua mente. La salvezza, l'unica ancora possibile, stava nel recupero, per intero, del femminile, un femminile nuovo, inedito, sapiente e mediatore, cosciente della forza e della bellezza del mondo, ma anche del magma selvaggio che attraversa la natura umana. Gor'kij si esprime apertamente per un ritorno in forze del matriarcato di cui era stato cultore fin dai tempi della sua polemica col filosofo Nikolaj Fëdorov: "L'uomo è finito in un vicolo cieco, non solo sociale, ma spirituale." cito da una lettera - "Quello che io auspico, ve lo dirò in breve, è un inevitabile ritorno al matriarcato. E' ormai chiaro che tocca alla donna farsi carico del compito di salvare l'umanità". Ma Marina muore e la sua morte improvvisa e violenta si porta via ogni speranza di rinascita e ogni risorsa vitale. Del resto La vita di Klim Samgin è un romanzo triste, senza prospettive, senza futuro, il futuro c'è già stato e s'è visto com'è andata a finire. Gli avvenimenti rotolano pesantemente verso la loro meta, mediati solo dalla coscienza del personaggio inconsistente, e per questo misterioso, che Gor'kij si è scelto come controparte della sua narrazione.

"Presto avrò 40 anni." - riflette Klim Samgin a buon punto del romanzo - "E' più di metà d'una vita. Sin da quando ero bambino mi venivano attribuite doti eccezionali. Per tutta la vita ho provato una santa insoddisfazione per gli uomini, per me stesso. Quest'insoddisfazione può essere soltanto il segno di una grande forza spirituale ${ }^{8}{ }^{\prime}$ ' in realtà

7. Gor'kij i sovetskie pisateli - neizdannaja perepiska, "Literaturnoe nasledstvo", Mosca 1963, v. 70, p. 593.

8. M. Gor'kij, La vita di Klim Samgin, Editori Riuniti 1962, parte IV, p. 160. 
il segno della sua nevrosi, di una paralisi spirituale che gli impone di cercare negli altri solo debolezze e negatività, nello sforzo di neutralizzare le proprie, in un circolo vizioso senza crescita né scampo.

Tutto era cominciato molto presto, nell'infanzia, perché nella sua famiglia di buone tradizioni intellettuali e populiste era applaudito quando inventava qualcosa di originale. Era stato programmato per essere un intellettuale raffinato a servizio del popolo. E difatti lo diventa, ma sarà un rivoluzionario controvoglia e comunque solo finché sarà bon ton esserlo. (Molto meno dopo la rivoluzione del 1905, quando il clima sarà cambiato ed egli non mancherà di adeguarsi). Comunque da bambino, siccome le sue trovate estrose erano molto apprezzate dagli adulti, egli passava intere giornate a escogitare frasi a sensazione, aggregazioni verbali insolite, a inventarsi, per compiacere gli altri e stupirli. Questo tratto della vydumannost' gli rimarrà sempre attaccato. Klim non sarà mai un uomo che cercherà se stesso, come nei romanzi di formazione, come nella splendida autobiografia, dove il personaggio forgia la propria personalità a contatto e in conflitto con gli altri, col mondo, con le difficoltà. Klim non diviene, perde il suo tempo, perderà tutta la vita a inventarsi, incoraggiato da quell'antica spinta, divenuta poi istinto, a impegnare tutte le sue risorse nella creazione di una maschera.

Ma se la famiglia assecondava in lui questi artifici coltivando, senza saperlo, la sua insincerità, l'egocentrismo, l'infelicità, con i coetanei questi trucchi non funzionavano. Lo chiamavano il sapientone e non lo consideravano. "Sono invidiosi" - tentava lui di difendersi, ma ne soffriva. Il suo cruccio era un ragazzo, Boris Varavka, il capobanda che godeva di naturale autorevolezza presso gli altri e aveva un talento particolare nello smascherare Klim e demolire le sue strategie per affermarsi.

Proprio un evento tragico legato a questo Boris segnerà il punto di rottura fra Klim e la sua coscienza. I ragazzi, un giorno, erano andati a pattinare sul fiume ghiacciato. Klim spiava in disparte il suo nemico nella speranza di vederlo cadere e renderlo ridicolo di fronte agli altri. Il ragazzo cade davvero e in un punto maligno, dove il ghiaccio è sottile. Precipita nel gorgo, cerca disperatamente di aggrapparsi ai bordi che si frantumano sotto le sue dita convulse. Klim vede tutto, accorre, si sfila la cintola e la lancia in soccorso, ma al momento buono, quando il ragazzo si aggrappa frenetico, Klim chiude gli occhi e molla la presa. Quando li riapre Boris è sparito. Succede tutto in un baleno, arrivano delle persone, arriva un contadino con una pertica per rovistare nell'acqua e Klim lo sente chiedere: "Ma c'era davvero quel ragazzo? O forse 
non c'era?". ' C'era, avrebbe voluto gridare Klim, ma non lo fa. Opta per la rimozione e accoglie in profondità quel suggerimento a nascondersi e a negare, innanzitutto a se stesso. Il contenuto di quella rimozione continuerà però a farsi vivo negli anni e per tutta la vita. Ogni volta che la coscienza lo chiamerà in causa per un'assunzione di responsabilità o per attentare alla sua fredda quiete di superficie ricorrerà a quello schema autoassolutorio per seppellire le obiezioni. "C'era davvero quel ragazzo? Forse non c'era" diviene il leit motiv della sua esistenza, quasi la precondizione a una potenziale carriera di delatore, carriera su cui fra l'altro insiste Romain Rolland (rispondendo con una lettera di commento al manoscritto inviategli da Gor'kij nel 1933). Comunque Klim si condanna a una vita di apparenze, così come gli era stato proposto nell'infanzia. Lascia che il sistema etico-culturale cui era stato esposto all'inizio della vita detti, senza intralci, la sua condotta di giovane e di adulto. Ma per procedere su questo binario è costretto a negare negli altri quello che nega in se stesso: non esiste alcuna personalità, gli individui sono solo un aggregato di pensieri di comodo e di principi provvisori. Le differenze fra le persone sono tutte nell'apparire, è lì che bisogna organizzarsi con un buon sistema di frasi da elaborare e da indossare come un abito. Sta dunque continuamente all'erta per smascherare il bluff con cui gli altri schermano il loro vuoto ed eventualmente anche per scippare loro qualche buona locuzione da esibire nei momenti giusti.

Ma Klim è anche un uomo intelligente, analitico, non è un caso che grossi episodi storici siano filtrati dalla sua vista nitida e perspicace. E' attraverso i suoi occhi quasi cinematografici che seguiamo passo a passo la grande manifestazione del 9 gennaio 1905, data memorabile in cui si rompe il patto secolare fra lo zar e il suo popolo, nell'insensata carneficina con cui lo spaventato Nicola II tenta di arginare gli spettri della sua rovina. Gor'kij si fida dello sguardo di Klim , sempre presente alle adunate di massa, frequenti e oceaniche all'epoca, forse perché la messa a fuoco non è qui volta all'interno, all'intimo, al profondo, dove la nevrosi di Klim non viene mai a capo della propria barriera narcisistica. Ricordo che il suo stesso cognome porta il marchio di questo eterno, fatale ripiegare su se stesso. Ogni tanto, tuttavia, il sospetto che gli altri abbiano dentro di sé qualcosa che a lui manca, una convinzione

9. M. Gor'kij, La vita di Klim Samgin, Einaudi, vol. 1, pag. 91. 
profonda, un centro segreto, fecondo di pensieri emotivi, lo assale, gettandolo nello sconforto. "Lui sapeva sviluppare alla perfezione le idee altrui, suffragandole da gran numero di citazioni, perché le risorse della sua memoria erano inesauribili. Ma sentiva bene che le sue cognizioni non si strutturavano in un sistema armonioso, non erano cementate da un'idea fondamentale". ${ }^{10}$ Eppure non arriva a cambiare, non sa o non può, nonostante il suo inconscio continui a non lasciarlo in pace, a segnalargli con i suoi messaggi quanto sia vulnerabile l'uomo che ignora tutto della propria vita interiore. Emblematico è il suo sogno dell'ombra. "Samgin si vide in una strada deserta, fra due filari di vecchie betulle. Accanto a lui camminava un altro Samgin. Era una giornata limpida, il sole scottava la schiena, ma né Klim, né il suo sosia, né gli alberi gettavano ombra e questo era molto preoccupante. Con la spalla il sosia spingeva Samgin verso le buche e i solchi della strada, lo spingeva verso gli alberi, gli intralciava il passo al punto che anche Klim gli diede uno spintone. Allora il sosia cadde a terra, si aggrappò alla gamba di Samgin e gettò un urlo selvaggio. Per non perdere l'equilibrio Samgin afferrò il suo compagno di viaggio, lo sollevò e si accorse che, come un'ombra, non pesava nulla. Eppure era vestito allo stesso modo di Samgin vivo e vero, quindi doveva avere un peso. Samgin lo sollevò da terra e lo scagliò lontano. Il sosia si ruppe in tanti pezzi e subito intorno a Samgin sorsero decine di figure in tutto simili a lui; lo circondarono, si misero a correre all'impazzata e sebbene fossero tutte senza peso lo urtavano, lo spingevano. Diventarono sempre più numerose e Samgin soffocava in mezzo a quella folla muta e silenziosa" ${ }^{11}$ Radiografia di una foresta di vuoti, dove Klim finisce a un certo punto della vita adulta, per altro socialmente ben integrata, ma non sa più trovare la strada del ritorno a sé per andare avanti; la sua disgrazia più grande è infatti il non essere in cammino, l'aver perso il vantaggio di poter considerare gli altri come individui e la possibilità di instaurare relazioni autentiche. Il risultato è una condizione di immaturità egocentrica che cerca solo conferme alla propria immagine fantastica. La sua mente intasata da cumuli di notizie, letture, incontri, avvenimenti, giudizi rimane separata dai sentimenti. Tutto è uguale a tutto, senza risonanza interna, e senza utilità. Si accorge di essere spettatore della vita senza aver vissuto. Ma continua a

10. M. Gor'kij, La vita di Klim Samgin, Editori Riuniti, parte IV, p. 356.

11. M. Gor'kij, La vita di Klim Samgin, Einaudi, vol. II, p. 1348. 
barare. E' pomposamente convinto di avere opinioni molto originali su tutto, in realtà si lascia trascinare dalle opinioni nell'aria come uno zimbello, mai padrone di ciò che pensa. Parla della guerra contro i tedeschi, ovviamente la prima guerra mondiale: "Non aveva ancora riflettuto seriamente sulle cause di quella guerra, ma si accorgeva che parlandone gli si infiammava un sentimento di ostilità verso di loro. Non aveva mai provato tale sentimento ed era turbato da ciò che giaceva nascosto in lui, che covava in lui da qualche parte e improvvisamente era esploso. Si ascoltava parlare e ascoltandosi si convinceva di quello che diceva". ${ }^{12}$ La stessa cosa avviene nei riguardi degli ebrei: "Prima era indifferente nei loro confronti, l'affare Bejlis comprometteva il Paese e il volto del Paese era la sua intelligencija. Era convinto che il suo atteggiamento nei riguardi dell'antisemitismo del governo fosse quello della maggioranza degli intellettuali. Ma da quando dal fronte era partita un'onda di odio animalesco verso gli ebrei, aveva pensato: "In effetti perché gli ebrei occupano posti così importanti da noi??. E poi: "Nella persona di Karl Marx l'ebraismo sparge sulla terra il suo distruttivo insegnamento sull'inconciliabilità di interessi fra capitale e lavoro, sull'inevitabile catastrofe rivoluzionaria...". ${ }^{13}$ Anche le sue idee fra virgolette rivoluzionarie vanno a farsi benedire, accodandosi rapidamente ai pregiudizi che sente in giro e spaccia come opinioni lungamente maturate. In definitiva Klim non è altro che l'uomo medio, come dice Musil: "Portatore dei bacilli di tutti gli orrori del mondo". ${ }^{14}$

Difficile collegare il Gor'kij che abita l'inconscio del suo personaggio per tutta la lunghezza del romanzo col Gor'kij univoco, compatto, semplificato che è stato tramandato. Il torto più grande che gli è stato inflitto è quello di aver lasciato sprofondare la sua opera a tutto vantaggio della figura pubblica, sicuramente singolare, ma che ha vissuto sempre in disparte, se non in autonomia rispetto a quella artistica. Gor'kij scrittore è rimasto sino alla fine libero ed è qui che occorre cercare il suo pensiero, i suoi enigmi, la sua vera voce. Si è tentati infatti di interpretare questo romanzo anche come risposta al duro antiumanesimo che si era instaurato, come una difesa a tutto campo dell'individuo quale che esso sia. Quando uno dei personaggi del romanzo più seguiti

12. M. Gor'kij, La vita di Klim Samgin, Editori Riuniti, parte IV, p. 407.

13. M. Gor'kij, La vita di Klim Samgin, Editori Riuniti, parte IV, pp. 497-498.

14. R. Musil, Diari, Eianudi 1980, vol. I, p. 673. 
e autorevoli, Makarov, commenta a proposito dei bolscevichi: "Per loro non esiste un problema morale, loro hanno la loro morale, o piuttosto, come dire, un sistema di igiene biosociale...parlare con loro dell'uomo, dell'individuo è del tutto inutile...l'uomo viene dopo dicono...dopo quando? Quando sarà arato il terreno per la sua libera crescita...". ${ }^{15}$ Gor'kij ha tutta l'aria di condividere in pieno la sua indignazione.

L'uomo ha certo bisogno sempre di qualcosa che lo liberi dal senso del precario e dell'inutile e cerca rifugio in mille forme di assoluto, nelle ideologie e nel fanatismo, ma le sorgenti dei suoi comportamenti vanno ricercate in ambiti diversi da quelli immediatamente visibili. Per sapere qualcosa di lui bisogna addentrarsi in altre zone più oscure e caotiche, dove si mischiano sostanze contrastanti, da cui non è semplice distillare gli elementi decisivi.

E' un po' il messaggio di fondo di Gor'kij, la celebrazione qui della sua posizione esistenziale.

La vita di Klim Samgin è un romanzo sul senso della vita, la ricerca religiosa, le sette russe, il matriarcato, l'idea gnostica dell'uomo, organo pensante della natura, non certo il testo fondante del realismo socialista, semmai il suo contrario, visto che nasce come genere tutto in negativo, come biografia di un uomo che, a giudizio stesso dell'autore, non merita alcuna biografia, ma a questa storia russa che è poi la non storia di un uomo inutile Gor'kij ha legato le sue riflessioni più intime, quelle che voleva fossero di lui tramandate. Lo considerava un romanzo testamento: "Non posso non scrivere La vita di Klim Samgin" - spiegava a Valentina Chodasevič - "e non ho il diritto di morire senza averlo fatto. All'inizio questo romanzo non sarà capito da nessuno, poi sarà trattato male e già lo è. Ma fra 50 anni si dirà: "C'è stato uno scrittore, Maksim Gor'kij, ha scritto molte cose e tutte molto brutte, ma se qualcosa di lui rimane è La vita di Klim Samgin". ${ }^{16}$

A parte questa osservazione un po' da melodramma, per il resto ha avuto ragione. Al suo primo apparire il romanzo non piacque a nessuno: agli émigrés parve un giudizio ingiusto sull'intelligencija troppo vanesia e chiacchierona, ai sovietici un insufficiente smascheramento di quella stessa intelligencija.

Oggi, liberati dall'ipnosi dei pregiudizi e dall'obbligo di cercare in-

15. M. Gor'kij, La vita di Klim Samgin, Einaudi, vol. II, p. 1255.

16. V. Chodasevič, Takim ja znala Gor'kogo, in "Novyj mir", 1968, n. 3, p. 58. 
dicazioni programmatiche "possiamo tranquillamente, con occhi liberi, guardare Gor'kij discutere con se stesso”, come scrive Suchich nel suo bel libro Zabluždenie ${ }^{17}$ i prozrenie Maksima Gor'kogo, e riaccoglierlo per intero nell'unico grande fiume degli scrittori russi da cui era stato tenuto per troppo tempo separato.

17. S. I. Suchich, Zabluždenie i prozrenie Maksima Gor'kogo, Nižnij Novgorod 1992. 